\title{
THE WHOLE- AND FRACTIONAL-COLONY MUTATION INDUCED BY SOFT X-RAYS IN YEAST ${ }^{1)}$
}

\author{
TERUTOSHI YAMASAKI, TAKASHI ITO \\ AND YORIAKI MATSUDAIRA
}

Biophysics Laboratory, Department of Physics, Rikkyo

(St. Paul's) University, Tokyo, Japan

Received June 30, 1964

Ito et al. (1962, 1963a, 1963b) and Yamasaki et al. (1963) reported that in diploid yeast cells, two types of mutation, fractional- and whole-colony, probably corresponding to the single- and double-strand change of DNA molecule, are induced by various mutagenic agents, that is, high temperature, UV, and chemicals such as hydroxylamine and acridine orange plus visible light. By X-rays, similar production of fractional- and whole-body mutations in Drosophila were reported by Altenburg and. Browning (1961), Matsudaira et al. (1964) and Inagaki (1962). The present short paper reports evidence for the two types of induced mutations in yeast by soft X-rays.

The yeast used in this experiment, a diploid strain of Saccharomyces cerevisiae heterozygous for adenine locus, has been described in a previous paper (Ito et al. 1962). This diploid was white in color and adenine non-requiring. An alteration in the $a d^{+}$gene yields either a wholly pink colony or a sectored pink colony.

Non-budding small cells gathered by slow centrifugation from the logarithmic phase culture, were irradiated on the surface of an agar plate by a low voltage X-ray tube operated at $39 \mathrm{kV}, 10.2 \mathrm{~mA}$ with $0.021 \mathrm{~mm} \mathrm{Ni}$ filter. The target-specimen distance was $12.5 \mathrm{~cm}$. Under these conditions photons should have nearly monochromatic energy $(8.05 \mathrm{keV})$ and the dose rate was $1,745 \mathrm{r} / \mathrm{min}$, when measured by a Victoreen roentgen meter for soft X-rays (Model 651). After irradiation the cells were incubated at $30^{\circ} \mathrm{C}$ for $3 \sim 5$ days, and then colonies with pink cells were counted according to the classification of whole- and fractional-(sectored-)colonies as induced variants. Determinations of genetic constitutions of pink and white cells in fractional-colonies were made using a similar technique to that employed in the earlier work (Ito et al. 1963b).

The white portions of the induced fractional-colonies all showed non-requirement of adenine, while the remaining pink portions and whole pink colonies required adenine. Induced variants were stable on transfer insofar as they were checked. Thirteen colonies among the fractional-colonies produced by $43 \mathrm{kr}$ irradiation were analysed. The results obtained are summarized in Table 1. Each fractional colony

1) This work has been supported by a research grant from the Ministry of Education and by a grant from the Toyo Rayon Foundation for the Promotion of Science and Technology. 
Table 1. Genetic constitutions of isolates from thirteen sectored colonies produced by irradiated vegetative cells of genotype $a d^{+} a d^{-*}$

\begin{tabular}{ccc}
\hline \hline $\begin{array}{c}\text { Genetic constitution of sectored colonies } \\
\text { White portion } \\
\text { (phenotypically wild type) }\end{array}$ & $\begin{array}{c}\text { Pink portion } \\
\text { (phenotypically mutant type) }\end{array}$ & colonies observed \\
\hline$a d^{+} a d^{-}$ & $a d^{-} a d^{-}$ & 3 \\
$a d^{+} a d^{-}$ & $a d^{-} l a d^{*}$ & 6 \\
$a d^{+} a d^{-}$ & $a d^{-} a d^{-}$or $a d^{-l a d}$ & 1 \\
$a d^{+} l a d$ & $a d^{-} a d^{-}$ & 1 \\
$a d^{+} l a d$ & $a d^{-} a d^{-}$or $a d^{-l a d}$ & 1 \\
\hline
\end{tabular}

* $a d^{+}, a d^{-}$-adenine; lad-recessive lethal in the locus or in the immediate vicinity of the adenine locus.

consists of a pink clone and a white clone. The genetic constitutions of these clones were determined with respect to $a d^{-}$gene and recessive lethal (lad) in the locus or in the immediate vicinity of that locus. Homozygous wild type, which may appear by somatic recombination, were not discovered in the white clones from fractionalcolonies. This suggests that the alterations in ad locus by X-rays are certainly due to gene mutation (including the non-reciprocal recombination or gene conversion), $a d^{+} \rightarrow a d^{-}$or $a d^{+} \rightarrow l a d$, rather than to mitotic crossing over.

Figure 1 shows the mutation frequency as a function of the exposure dose, as classified into whole- and fractional-mutations. The curve of the whole-colony mutation frequency shows a multi-hit type. The yield of fractional-colonies increases with dose and levels off at the higher doses. And total frequency (whole + fractional) increases almost linearly with the exposure dose. Consequently the ratio of fractionalcolony to whole-colony decreases with increasing exposure dose. Surprisingly enough, these relationships between mutation frequencies and doses are very much like those which have been obtained previously with ultraviolet light (Yamasaki et al. 1963).

Thus essentially the same hypothesis may be advanced: The elementary process of mutation is a fractional-colony mutation (probably corresponding) to the singlestrand change of DNA molecule of a gene), caused by the first hit of X-rays; and an additional hit changes the fractional-colony mutation into a whole-colony one (which would correspond to double-strand change of DNA). If this interpretation is correct, it is easily anticipated that more fractional-colony mutations would be produced than the whole-colony mutations at sufficiently low dose; and as the dose increases more whole-colony mutations would appear because of the increased chances for receiving two hits. Furthermore, the fractional-colony mutations induced near the end of the exposure will not have as much chance of an additional hit for changing into the whole-colony mutations as those being induced earlier. Therefore the fractional-colony mutation frequency would level off at higher doses.

It has been reported by other workers (e.g., Alexander and Stacey 1955; Lett et al. 1961) that each strand of DNA molecule was broken down by ionizing radiation. 


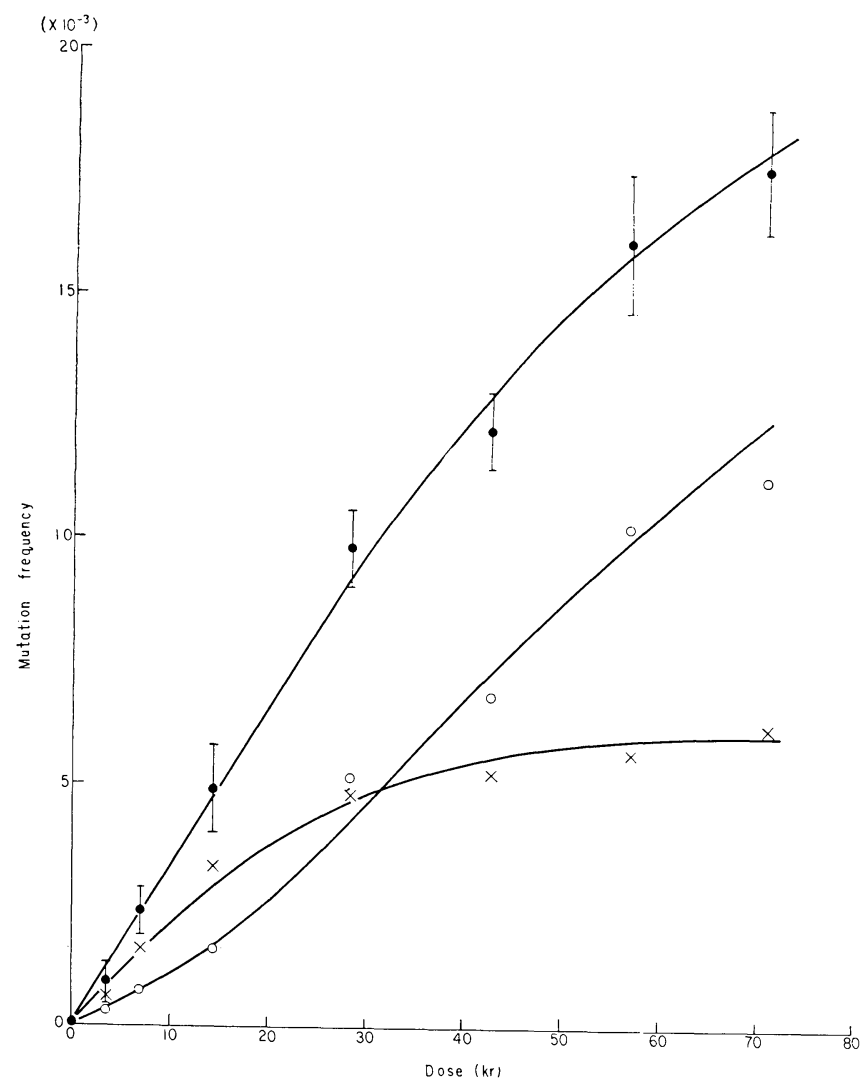

Fig. 1. Mutation frequency at $a d$ locus induced by soft X-rays in diploid yeast cells. $\bigcirc-\bigcirc$ : Whole-colony mutation, $x-x$ : Fractional-colony mutation, $\bullet-\bullet$ : tolal (whole + fractional) mutation.

Obviously it cannot be established in this experiment that the fractional- and wholecolony mutation, respectively, correspond to the single- and double-strand change of DNA molecule. The results, indicating multi-hit type in whole-colony production, are, however, consistent with the facts that the expected spacing of ionization by $8.5 \mathrm{keV} \mathrm{X}$-rays $\left(28.2 \mathrm{~m} \mu\right.$ in tissue of density $1 \mathrm{~g} / \mathrm{cm}^{3}$ [Lea 1947]) is well over the distance of the presumed two targets in the gene, thereby allowing only a very small chance of the ionization to affect both targets by a single track. Therefore, it is not inconsistent with the experimental results that the dual structure of a gene corresponds to the two strands of DNA molecule of a gene.

\section{LITERATURE CITED}

Alexander, P.A. and K. A. Stacey, 1955 Evidence from light-scattering studies for a dimeric structure for deoxyribonucleic acid in solution. Biochem J. 60: 194-200.

Altenburg, E. and L. S. Browning, 1961 The relatively high frequency of whole-body mutations compared with fractional induced by X-rays in Drosophila sperm. Genetics 46: 203-211. 
Browning, L. S. and E. Altenburg, 1961 Gonadal mosaicism as a factor in determining the ratio of visible to lethal mutations in Drosophila. Genetics 46: 1317-1321.

Inagaki, E., 1962 The comparison between whole and fractional mutation rate induced by X-rays in Drosophila. (Abstract). Jap. J. Genet. 37: 388.

Ito, T., T. Yamasaki and Y. Matsudaira, 1962 Studies on the genetic multiplicity of a gene in yeast cells. I. Characteristics of the induced mutation by subcritical temperature and ultraviolet light. Jap. J. Genet. 37: 276-283.

Ito, T., T. Yamasaki and Y. Matsudaira, 1963a Mutation induced by acridine orange plus visible light. Kagaku (Japanese) 33: 383-384.

Ito, T., T. Yamasaki and Y. Matsudaira, 1963b Mutation induced by hydroxylamine in yeast cells-Evidence for the occurrence of single-strand change. RUP-16 from Rikkyo University.

Lea, D. E., 1947 Actions of Radiations on Living Cells. Cambridge University Press, Cambridge, England.

Lett, J. T., K. A. Stacey and P. Alexander, 1961 Crosslinking of dry deoxyribonucleic acid by electrons. Radiation Research 14: 349-362.

Matsudaira, Y., T. Ito, T. Yamasaki, S. Ishizaka and M. Domon, 1964 On the fractional- and whole-body mutations induced by soft X-rays in Drosophila. Jap. J. Genet. 38: 288-289.

Yamasaki, T., T. Ito and Y. Matsudaira, 1963 Studies on the genetic multiplicity of a gene in yeast cells. II. Effects of ultraviolet light. J. Radiat. Research 4: 85-90. 